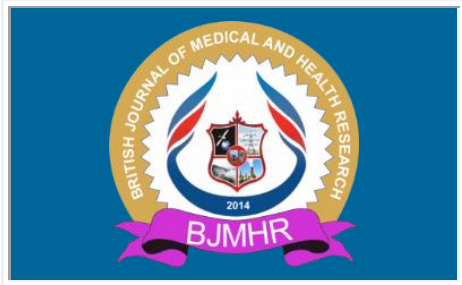

BJMHR

British Journal of Medical and Health Research

Journal home page: www.bjmhr.com

\title{
Spleenic Abcess: Rare Spectrum of Diagnosis In Immunocompetent Patient With Urinary Tract Infection
}

Anubhav Gupta*1, Jagrati Gupta ${ }^{2}$, Hakimuddin Pardawala ${ }^{\mathbf{3}}$

1. Department of Medicine, Saifee Hospital, Mumbai, Maharashtra

2. Department of Family Medicine, Saifee Hospital, Mumbai, Maharashtra

3. Head of Department of Medicine, Saifee Hospital, Mumbai, Maharashtra

\section{ABSTRACT}

Spleenic abscess is a rare entity, with a reported frequency of $0.05-0.7 \%$.Its reported mortality is still high, up to $47 \%$, and has the potential to reach $100 \%$ among patients who do not receive antibiotic treatment. It is more common in the presence of infection at different primary sites, especially endocarditis or in cases of ischemic infarcts that are secondarily infected. Immunosuppression and trauma are well-known risk factors. Recently, intravenous drug abusers and alcoholics have shown an increased incidence compared to other high-risk groups. However, encountering this entity in general population is uncommon. Clinical examination and laboratory findings are not constant; thus, imaging is a necessary tool for establishing the diagnosis, with a choice between ultrasound and computed tomography. If untreated, the mortality reaches almost $100 \%$. Treatment includes conservative measures and surgical interventions. Spleenectomy has been the preferred approach in most centers. More recent studies have suggested the use of advanced and alternative options, including laparoscopic surgical and percutaneous interventions. Changing trends, in view of the importance of immunological role of spleen, have emphasized more on spleen preserving protocols, especially in children and young patients, and in cases of solitary abscess with a thick wall. Conducted a literature review by analysis of various high-risk groups, presentation, diagnosis and treatment of splenic abscess and have presented a case report here. Spleenic abscess is an infrequent clinical entity and carries a very high mortality in the untreated cases. Pathognomic clinical features of left hypochondrial pain, splenomegaly, fever and leucocytosis are present in only one - third of the cases. Diagnosis is mostly done by imaging, either Abdominal Sonography or CT Scan. So far no prospective study has been done to rationalize the management, probably due to paucity of cases. Antibiotics alone or with percutaneous drainage under imaging control are successful in many cases. Failed aspirations and splenic abscesses with concomitant pathologies need spleenectomy. Spleenectomy and complete removal of the source of the infection is still the mainstay of treatment. Detailed review of the available literature of the clinical problem and various methods of treatment of spleenic abscesses are discussed in this article.

Keywords: Spleenic Abcess, Surgical interventions

*Corresponding Author Email: anubhavgupt99@gmail.com

Received 05 December 2020, Accepted 03 January 2021

Please cite this article as: Gupta A et al., Spleenic Abcess: Rare Spectrum of Diagnosis In Immunocompetent Patient With Urinary Tract Infection. British Journal of Medical and Health Research 2021. 


\section{INTRODUCTION}

A 84-year-old man presented to the medical IPD with complaint of intermittent high-grade fever with chills and rigors, which was associated with generalized weakness and vomiting containing food particles. Patient also c/o loose stool, watery in consistency 4-5 episodes which gradually subsided. Past h/o urinary tract infection 4-5 wks before with urine culture sensitivity showing no growth. No h/o abdominal pain, burning micturition, cough. On general examination, raised body temperature, dehydration present.

On systemic examination no specific findings. Initially managed with iv ceftriaxone, fever subsided but total leucocyte count remained at $19970 / \mathrm{cmm}$ that warranted further investigations.

Ultrasound of abdomen (31Dec18) showed spleen bulky in size with thick walled irregular marginated lesion with central anechoic area measuring $11.1 * 5.4 * 7.5 \mathrm{~cm}$ with a volume of $237 \mathrm{cc}$. In view of ultrasound report a computed tomography with contrast scan done (1Jan19) which shows partial thrombosis of splenic vein with extensive splenic infarction, few fibro calcific atherosclerotic plaques at the origin of superior mesenteric artery and both renal arteries, causing mild luminal narrowing . (Figure 1. A, figure 1.B \&figure 1.C))

We got controversial reports but a provisional diagnosis of splenic abscess was made after a repeat ultrasound which shows similar lesion as of previously scan and intravenous antibiotics were started. HIV, Widal test, malaria, dengue and urine culture came out to be negative and blood culture reveal E coli infection in blood. Infective endocarditis seems to be the most common cause of spleenic abcess. In view of this, transesophageal echocardiography done which was normal. Blood investigations were significant for the presence of leukocytosis $(19,000 / \mathrm{mm} 3)$ and with reactive thrombocytosis with a platelets counts of $602000 / \mathrm{mcl}$. Patient got intermittent fever spikes with leucocytosis up to 35000/mm3. Laproscopy was done in view of sudden raised total leucocyte count, ommentum adherent to spleen with foul smelling pus collection in the spleen.(figure 2) . Spleenectomy was done and his general condition improved uneventfully.

Patient was managed conservatively with intravenous antibiotics, ceftriaxone $2 \mathrm{gm}$, imepenem + cilastatin $(1.5 \mathrm{gm})$, meropenam(500 mg ), sulbactam( $500 \mathrm{mg})$, inj septran (480 $\mathrm{mg}$ ), iv fluconazole (500 mg ). He responded well to treatment, and symptoms resolved and counts decreased. Histopathology done of spleenic sections suggests extensive areas of necrosis along with dense inflammatory infiltrate composed of neutrophils, plasma cells and histocytes suggestive of ruptured spleen abcess. (Figure 1) 


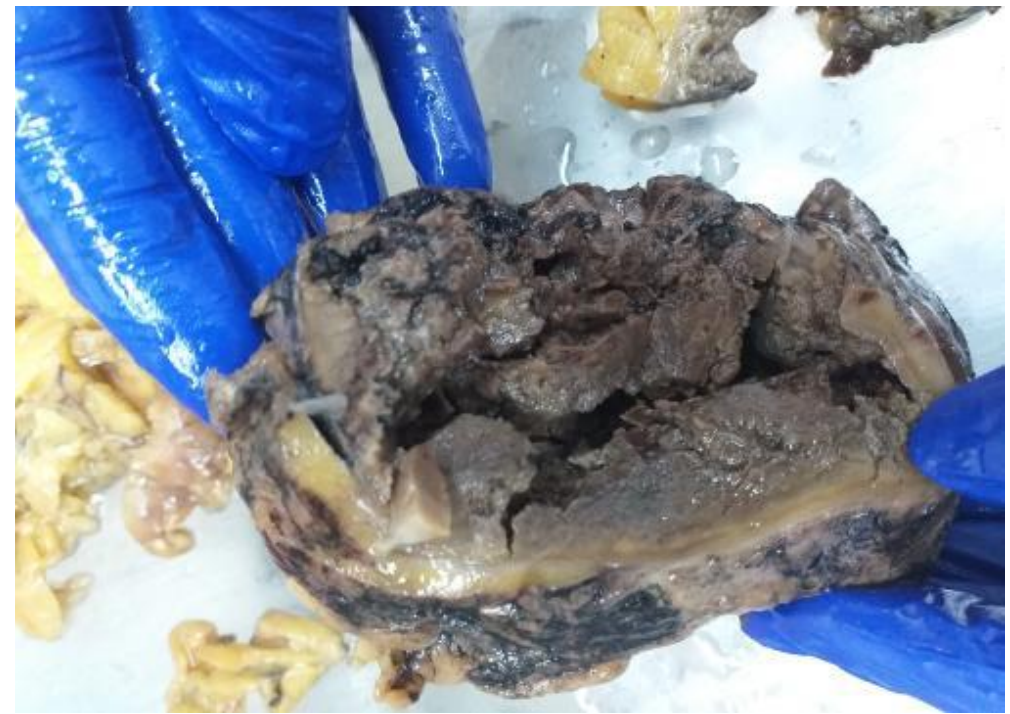

Figure 1

\section{DISCUSSION}

Splenic abscess is an uncommon entity among immunocompetent adults. As noted previously, the autopsy incidence of splenic abscess ranges from $0.1 \%$ to $0.7 \%$. Infective endocarditis, diabetes mellitus, trauma, intravenous drug abuse, and hemoglobinopathies are some of the reported predisposing factors in immunocompetent adults.

The spectrum of organisms that can cause splenic abscess is very large and has tended to change over time. An American study conducted nearly 3 decades ago found that anaerobes were more common as causative organisms than the aerobes; among the aerobes, E coli was the most common isolate which we get in this case also . Other studies published in the past 17 years also noted that gram-negative organisms were the most common causative organisms. However, a more recent study found that gram-positive organisms were more than twice as common as gram-negative organisms. Polymicrobial flora is responsible for at least $10 \%$ to $15 \%$ of patients with splenic abscess. Negative culture from splenic abscess have been reported in up to $30 \%$ of cases, which may reflect prior antibiotic use or fastidious organisms. Apart from these, cases of splenic abscess caused by Mycobacteria, Brucella, Coxiella burnetti, Bartonella and other organisms (eg, Candida and Actinomyces) have also been reported. Similar to other studies, our series also highlights a multitude of causative organisms. Collectively, these data point to the extremely diverse microbiology of splenic abscesses and suggest that establishing a microbiological diagnosis is of paramount importance.

Splenic abscess is an uncommon entity. The incidence of splenic abscess in various autopsy series has been estimated to be between $0.2 \%$ and $0.7 \%$. In one series of 540 intra abdominal abscesses none were in the spleen . However, this uncommon disease is recently being reported more frequently. Two main contributing organisms are found to be responsible for forming splenic abscess: Klebsiella pneumonia and streptococcus. 
Almost all splenic abscesses develop as a result of bacterimia ( or fungemia) although a small percentage spread to the spleen from a contiguous site .

Endocarditis is the most classical underlying condition that result in splenic abscess, although urinary tract infection, surgical wound infection, and gastrointestinal infection that can result in bacteremia also leads to splenic abcesses.

Two main contributing factors to the apparent increase in the incidence of splenic abscess are , first is advances in imaging studies with ultrasound being single most useful modality with sensitivity ranging $70 \%$ to over $90 \%$ and computed tomography scan is the most sensitive modality with greater than $90 \%$ sensitivity in most cases and second is, an increased number of immune compromised, trauma and cancer patients. Spleenic abscesses may often be misdiagnosed, because the signs and symptoms are non-specific; nevertheless, modern imaging has improved the process of their diagnosis.

In this series, treatment was carried out by antibiotics and percutaneous drainage or spleenectomy. The mortality rate appeared to be related to the patient's general underlying condition. Our patients recovered from their spleenic abscess regardless of the treatment modality. Thus, based on these observations, the prognosis for these patients cannot be accurately predicted by the intervention methods.

Percutaneous treatment of splenic abscess is an effective treatment alternative to surgery; furthermore, it offers the theoretical advantages of preserving immunologic function by avoiding splenectomy in young patients. The success rate of percutaneous drainage for spleenic abscess has been reported between $67 \%$ and $100 \%$. Historically, its initial use was in those patients thought unable to tolerate open surgery, but several series have shown that CT and ultrasound guided percutaneous aspiration have success rates comparable with open surgery with less initial morbidity and mortality.

Traditional treatment includes appropriate antimicrobial therapy with or without spleenectomy. There are a number of studies in favor of spleen preservation and management using percutaneous drainage. Thus percutaneous aspiration or drainage may be used as a bridge to surgery, allowing non-operative healing for spleenic abscess patients who are at risk for surgery, and helps avoid the risk of a fulminant and potentially life-threatening infection.

Removal of spleen is associated with an increase risk of sepsis due to uncapsulated bacteria, such as Neisseria meningitidis, streptococcus pneumoniae and Hemophilus influenzae and with intra erythrocytic parasite such as Plasmodium spp and babesia. A proper immunisation is required post splenectomy with influenza vaccine, penumococcal vaccine and Hib vaccine with maintenance of proper hygeine to avoid infection and their sources. 
In conclusion, the best therapeutic approach for splenic abscess is still a matter of debate. We agree with the traditional treatment of antibiotics with or without splenectomy rather than not treating splenic abscess which carries nearly $100 \%$ mortality.

However, based on our experience and the current literature, percutaneous aspiration of splenic abscess can be used as a bridge to surgery for those patients who are critically ill or who have several co-morbidities. Early diagnosis of spleenic abscess requires a high degree of suspicion. Most of our patients presented with unexplained fever, abdominal pain and radiologic evidence of pathology in the left chest or abdomen, and these signs and symptoms should lead to suspicion of splenic abscess. Abdominal CT and ultrasonography were usually diagnostic. We suggest that treatment should be tailored and applied to splenic abscess patients on an individual basis.

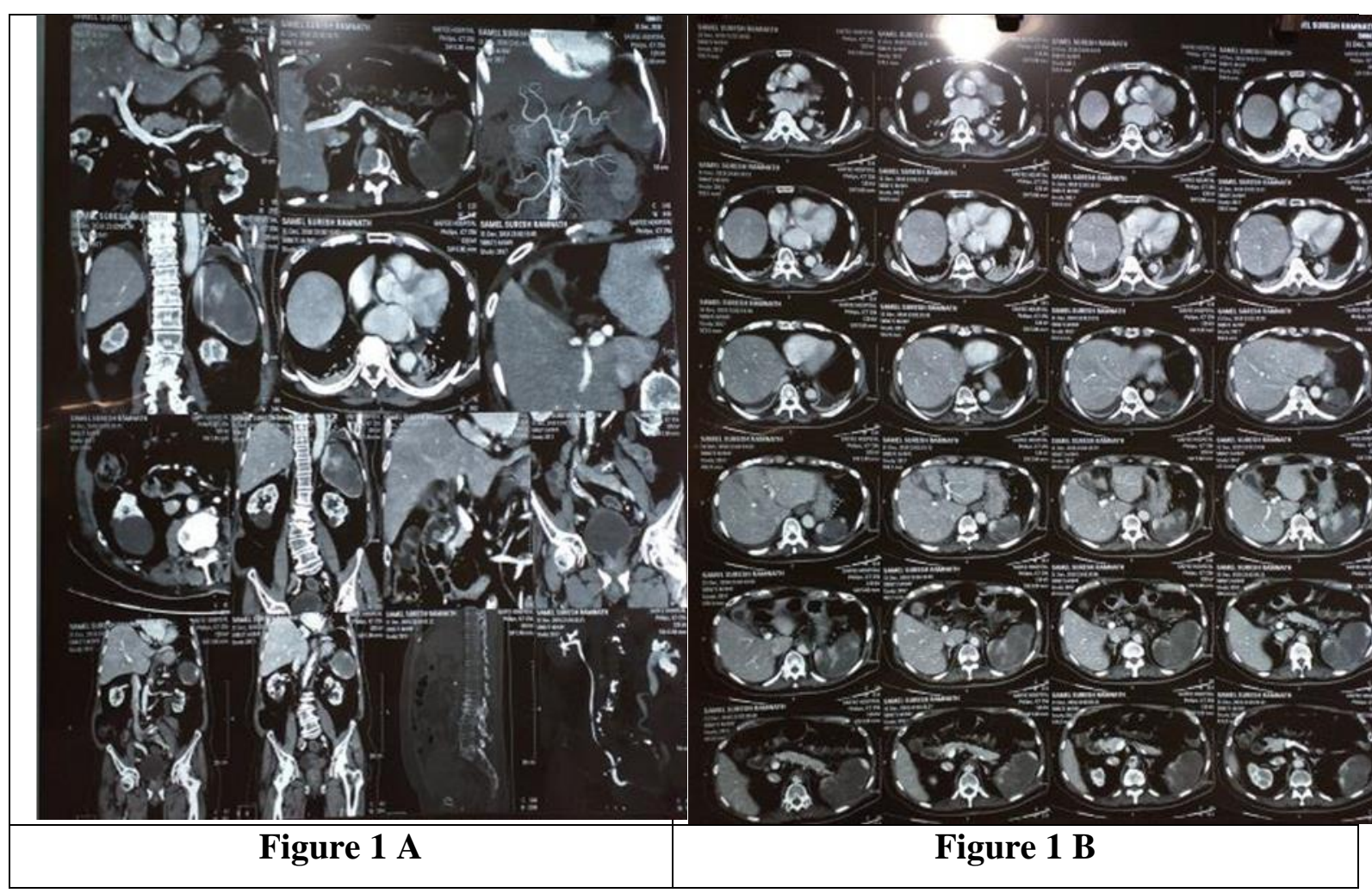




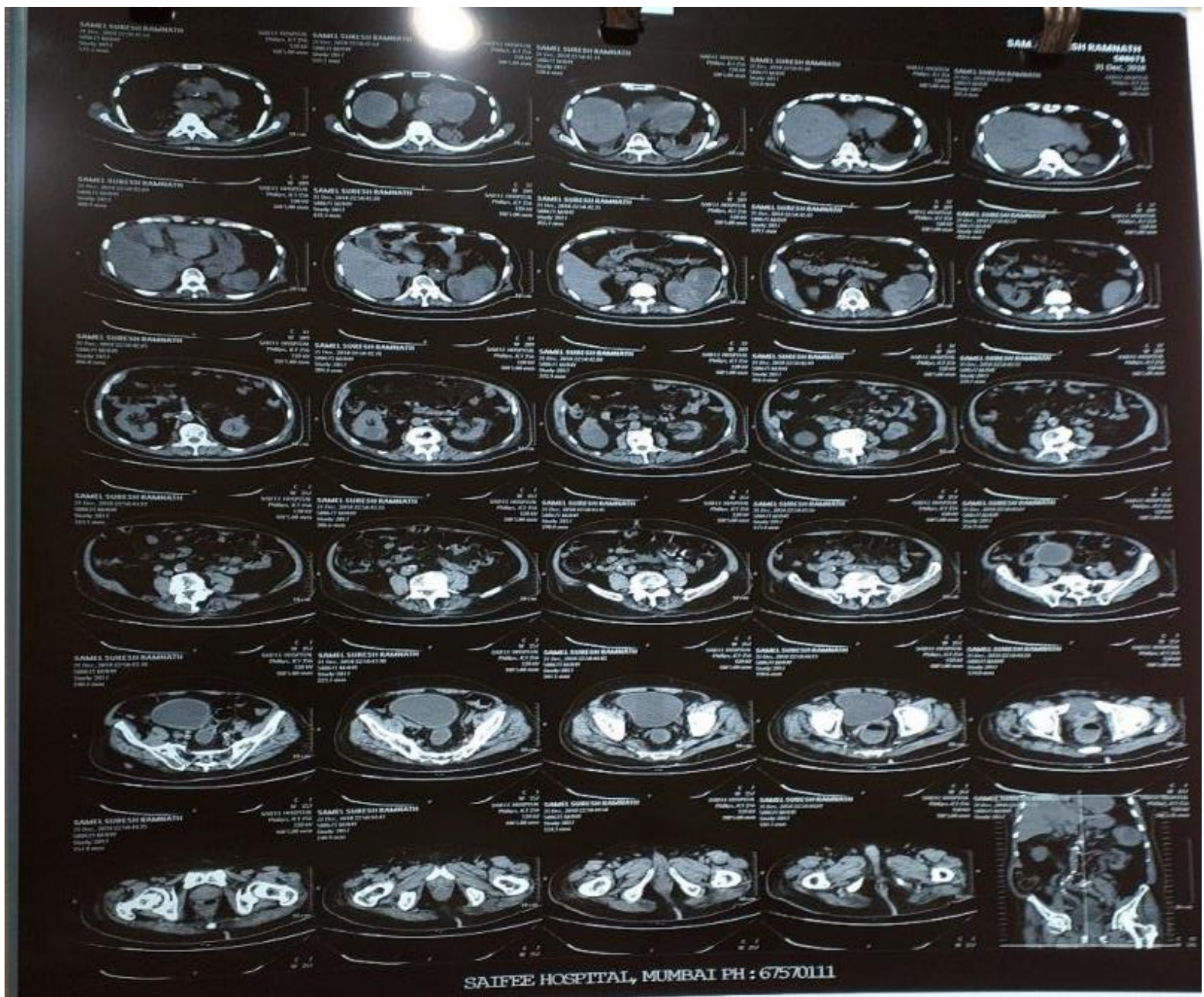

Figure 1.C

\section{REFERENCES:}

1. Ooi LL, Leong L. Splenic abscesses from 1987 to 1995. Am J Surg 1997;174:87-93.

2. Carbonell AM, Kercher KW, Matthews BD, Joels CS, Sing RF, Heniford BT. Laparoscopic splenectomy for splenic abscess. Surg Laparosc Endosc Percutan Tech 2004;14:289-91[PUBMED]

3. Chiang IS, Lin TJ, Chiang IC, Tsai MS. Splenic abscesses: review of 29 cases. Kaohsiung J Med Sc2003;19:510-5. [PUBMED]

4. Chulay JD, Lankerani MR. Splenic abscess: Report of 10 cases and review of the literature. Am J Med 1976;61:513-22.

5. Chang KC, Chuah SK, Changchien CS, Tsai TL, Lu SN, Chiu YC, et al. Clinical characteristics and prognostic factors of splenic abscess: A review of 67 cases in a single medical center of Taiwan. World J Gastroenterol 2006;12:460-4 [PUBMED]

6. Kim HS, Cho MS, Hwang SH, Ma SK, Kim SW, Kim NH, et al. Splenic abscess associated with endocarditis in a patient on hemodialysis: A case report. J Korean Med Sci 2005;20:313-5[PUBMED] 
7. Smith MD Jr, Nio M, Camel JE, Sato JK, Atkinson JB. Management of splenic abscess in immunocompromised children. J Pediatr Surg 1993;28:823-6[PUBMED]

8. Swamy TK, Balachandar TG, Chandramohan SM, Manohara G, Ali MA. Splenic abscess in a HIV patient. Trop Gastroenterol 1995;16:29-31. [PUBMED]

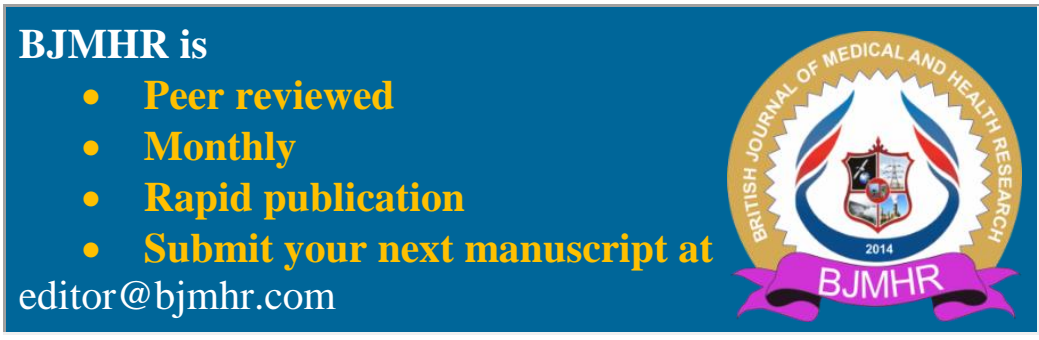

